\title{
FT-NIR: A Tool for Process Monitoring and More
}

\author{
Domenico Martoccia ${ }^{a *}$, Holger Lutz ${ }^{b}, Y_{v a n}$ Cohen $^{c}$, Thomas Jerphagnon ${ }^{c}$, and Urban Jenelten ${ }^{c}$
}

\begin{abstract}
With ever-increasing pressure to optimize product quality, to reduce cost and to safely increase production output from existing assets, all combined with regular changes in terms of feedstock and operational targets, process monitoring with traditional instruments reaches its limits. One promising answer to these challenges is in-line, real time process analysis with spectroscopic instruments, and above all Fourier-Transform Near Infrared spectroscopy (FT-NIR). Its potential to afford decreased batch cycle times, higher yields, reduced rework and minimized batch variance is presented and application examples in the field of fine chemicals are given. We demonstrate that FT-NIR can be an efficient tool for improved process monitoring and optimization, effective process design and advanced process control.
\end{abstract}

Keywords: FT-NIR $\cdot$ Real-time process analysis

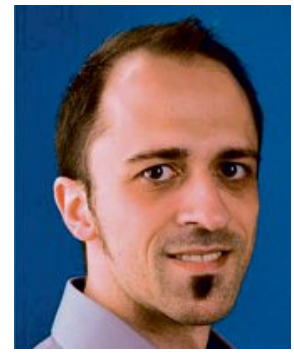

Domenico Martoccia studied Physics at the University of Zurich, Switzerland. He received his $\mathrm{PhD}$ in Physics from the Paul Scherrer Institute in Villigen, Switzerland by solving the structure of graphene depositions on different substrates. This work was done using the high performance of synchrotron radiation and the power of surface X-ray diffraction. In 2010, he was hired by Bruker as a sales representative for FT-IR/FT-NIR and Raman systems, and was promoted to country manager Switzerland in 2012. After a further promotion to Regional manager in 2014, he has taken on the additional responsibility for the sales teams in Austria, Italy, France, a task that he enjoys and is fully engaged in.
${ }^{\star}$ Correspondence: Dr. D. Martoccia ${ }^{a}$

E-mail: domenico.martoccia@bruker.com

aBruker Optics $\mathrm{GmbH}$

$\mathrm{CH}-8117$ Fällanden

${ }^{\mathrm{b} B r u k e r}$ Optics $\mathrm{GmbH}$

76275 Ettlingen, Germany

'Firmenich SA

$\mathrm{CH}-1283$ La Plaine

\section{Introduction}

Process monitoring and control in the fine chemical industry is today still mostly limited to the measurement and control of physico-chemical parameters like temperature, pressure, density, conductivity, $\mathrm{pH}$ or $\mathrm{pO}_{2}$. Information on a molecular level typically provided by gas chromatography (GC) or high-performance liquid chromatography (HPLC) is mostly only available in off-line mode (see Fig. 1). The traditional off-line (instrument located in a laboratory) or at-line (instrument located in a production area) modes require a sample which is normally taken manually. This sample is then analyzed by a technician in a laboratory (off-line) or by an operator with the instrument located in the production area (at-line). It takes typically 30-60 minutes for the result to be available. The

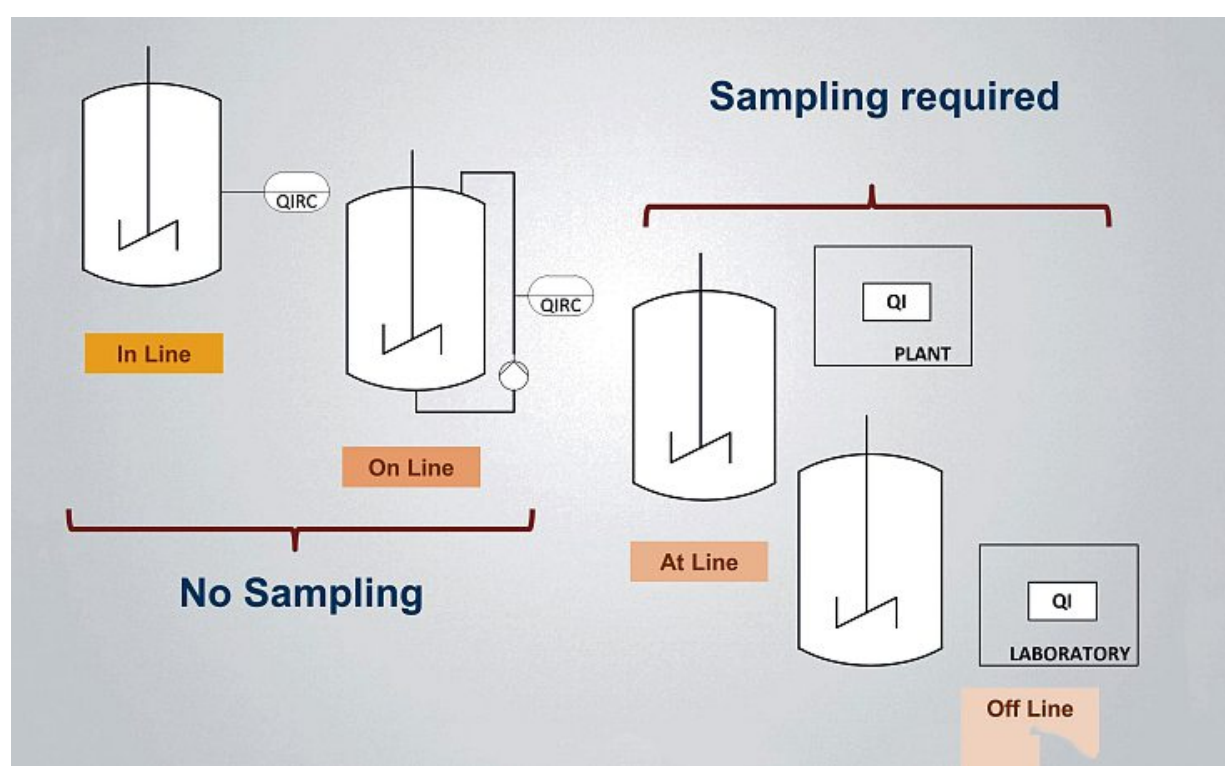

Fig. 1. Different production analysis principles. whole process being rather labor intensive clearly limits the potential sampling and measurement frequency. The risk of contaminations of both the sample as well as the process and in many cases the need for sample quenching and/or stabilization are further drawbacks. For fed-batch processes off-line/at-line analysis often requires an interruption of the process leading among other consequences to an increased batch cycle time.

These drawbacks call for in-line or online measurement technologies providing information on the molecular level at the point of interest in real time without the need for sampling. In the case of in-line measurements, the probe is directly placed in the vessel providing real time results at a high frequency. On-line measurement tools are often placed in a recirculation loop allowing a variety of pre-treatments before 
analysis, leading to a short delay. Both inline and on-line instruments are less prone to human error and typically directly linked to the process control system for data visualization and recording. At the same time, the results can be used without delay for process control purposes. The last decades have seen significant innovation and development in the field of in-line/on-line instrumentation and today a range of spectroscopic tools including FT-IR (Fourier Transform Infrared) and FT-NIR (Fourier Transform Near-Infrared) are available. These methods allow fast and accurate inline sensing of key parameters including the concentrations of substrates as well as main and side products leading to comprehensive reaction monitoring and ultimately process control. These non-destructive techniques provide a multi constituent analysis with accuracies similar to currently used (offline) methods (i.e. GC, HPLC, NMR).

\section{Spectroscopic Technology}

\subsection{FT-NIR - General Aspects}

Today FT-NIR spectroscopy is increasingly gaining popularity as an important method for monitoring of chemical processes. ${ }^{[1]}$ A growing number of companies complement or replace conventional analytical tools with spectroscopic methods which yield analytical results within just a few seconds. The main driver of these efforts is always the potential to reduce costs and increase competitiveness. Avoiding time-intensive off-line analysis often enables further optimization of production processes.

The installation of NIR-systems is rather straightforward: It only requires introducing a probe or sensor head in the process via a standard measurement port. The measurement signal is transmitted to the spectrometer via an optical fiber. The spectrometer, which performs the measurement, is connected to the PC, where the evaluation is done. It transmits the results via a communication interface to the process control system (see Fig. 2).

By virtue of this technique, to transmit the signals via light, the spectrometer does not need to be installed adjacent to the production vessel as it would be the case for FT-IR installations, thereby avoiding costly investments for temperature and humidity control, explosion prevention, etc. Furthermore, besides the light source which needs to be changed annually, a spectrometer requires almost no consumables causing therefore only negligible operational and maintenance costs.

\subsection{FT-NIR Application Fields}

With one single NIR measurement of the absorption of near-infrared light of the

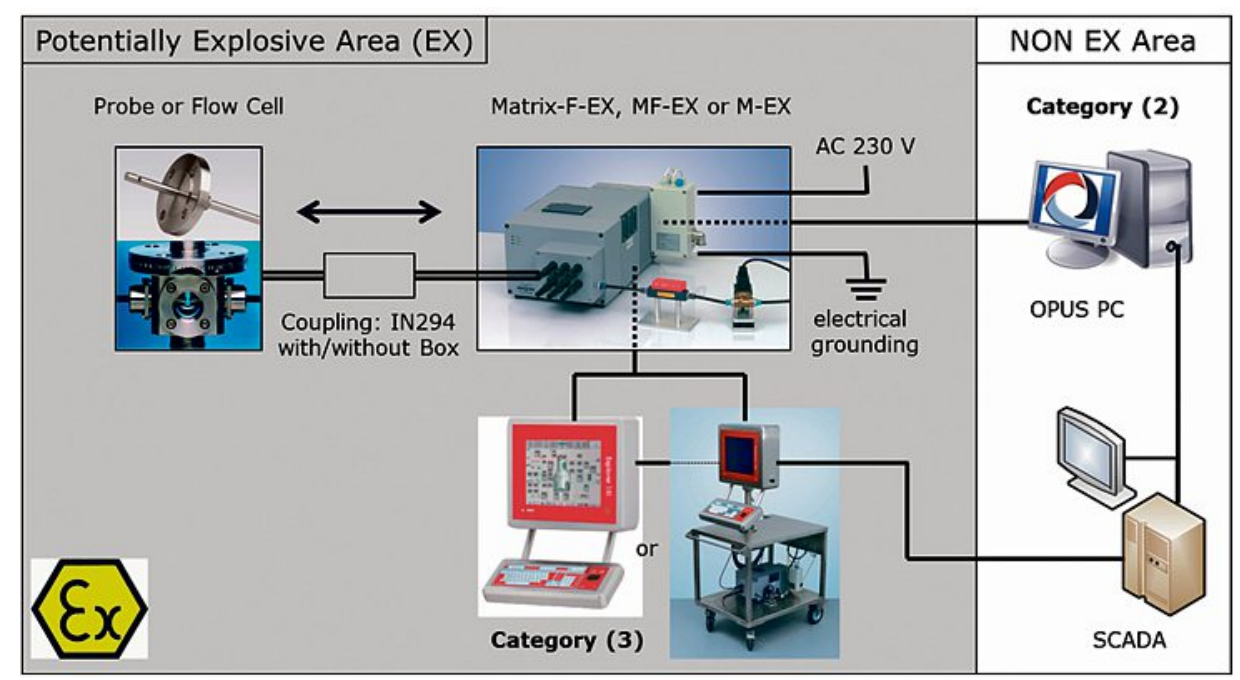

Fig. 2. Schematic view of a Bruker Matrix-F installation, where the system itself is outside the potentially explosive zone (EX) and only the fibers and the probes are inside the EX-zone.

sample at different wavelengths, multiple components can be analyzed in less than one minute in solid samples as well as in liquids. The recorded NIR spectrum is characterized by overtones and combinations of the fundamental molecular vibrations of molecules containing $\mathrm{C}-\mathrm{H}, \mathrm{N}-\mathrm{H}$ or O-H groups, making NIR spectroscopy the first choice for the analysis of organic materials in the chemical, pharmaceutical, food, feed and agricultural industries. This opens a wide field of applications, making FT-NIR a valuable tool for raw materials inspections, in-process-monitoring/ control as well as final quality testing and validation. ${ }^{11]}$

\subsubsection{Raw Materials Identification}

Every organic material has a unique NIR spectrum - like a finger print. Therefore, NIR is widely used as a reliable tool for substance identification and authentication, especially for inspection of incoming raw materials. Fully automated software routines provide quick information whether a tested raw material is identical to the expected substance (identification) and whether it meets its required specifications (authentication). Along the same lines, FT-NIR is also a powerful and efficient tool to verify that the raw materials charged into a vessel correspond well to the established recipe, allowing to identify again off specification material, raw material concentration variations and contaminations as well as preparation and charging errors.

\subsubsection{Multi Component Quantitative Analysis}

The amount of light absorbed by the sample depends on the concentrations of its ingredients (Beer's Law). In order to quantify the different components of an ingredient mixture the spectrometer determines the light absorption/loss at the related characteristic frequencies. Since the NIR spectrum contains the analytical information of every component of the mixture simultaneously, quantitative information of all constituents is accessible by collecting just one spectrum, once the system has been calibrated for the individual components. Data generated by this approach can be used by the production personnel for real-time decisions. Regardless whether the instrument is delivered with established calibrations or if the users create their own calibrations, a fast analysis of multiple components is provided in an efficient and cost-effective manner.

\subsubsection{In-line Process Control}

The fiber optic probes allow the point of analysis to be located directly in the reactor or tank/container. Typical examples are the monitoring/surveillance of chemical reactions, distillations and rectifications, crystallizations and drying processes. Specific quality parameters can be controlled in real-time directly in the process, rendering time-consuming lab analysis superfluous. Since results are obtained within a few seconds, the process can be followed with high accuracy in real time. This allows in many areas to directly control the production processes on a molecular level via FT-NIR spectroscopy, leading to increased yield, reduced batch cycle times, better product quality and fewer out-of-spec batches.

\subsubsection{Process Understanding and Inno- vation}

Spectroscopic analytical technology providing real-time information on a molecular level is also very useful to gain insight into the reaction kinetics as well as understanding separation processes. These devices allow process knowledge to be gained in a fast and efficient manner compared to the typically used, low frequency standard methods (GC and HPLC). This 
is very useful during the process development phase but also later for process innovation and process optimization on a production scale.

These analytical tools can also play a pivotal role in the identification of the key process parameters and their evolution towards the 'Golden Batch' (i.e. the ideal batch providing both an excellent yield, productivity and final product quality). Once the characteristic profile of a 'Golden Batch' has been identified, spectroscopic tools can also serve as a vector to put recipes and control algorithms in place to replicate the selected key process trajectories on a regular basis.

\subsection{Advantages of FT-NIR Spectroscopy}

Compared to classical physico-chemical, wet chemical and chromatographic analytical tools FT-NIR technology is quick, cost-effective, non-destructive, and safe as no hazardous chemicals are used. Further advantages include:

\subsubsection{Precision in a Production Envi- ronment}

Bruker's patented 'Rock-SolidInterferometer' uses Cube Corner mirrors, in contrast to flat mirrors which are used in the classical 'Michelson Interferometer' (Fig. 3). Cube Corner mirrors guarantee an ultra-precise guidance of the light beams passing the interferometer, even if external vibrations occur. Especially in a chemical industrial production environment, this is of crucial importance.

\subsubsection{Direct Calibration Data Transfer}

The FT-NIR technology provides a high wavenumber accuracy and permits the transfer of calibration models from one FT-NIR instrument to another without time-consuming standardization routines. A stable calibration can be run for many years or even decades without having to be adjusted.

\subsubsection{Easy and Accurate Analysis of Heterogeneous Samples}

Since NIR methods require no sample taking and preparation, the potential for sampling error is significantly reduced, leading to an improved analytical accuracy and reproducibility. This also eliminates the contamination risk both for the sample and the process. For an NIR measurement in the lab, the sample is simply filled into a glass vial or Petri dish as glass is transparent in the NIR spectral region. Since NIR light not only analyses the surface but penetrates deeper into the material (several micrometers to millimeters), it is ideally suited for the analysis of heterogeneous samples leading to much more reliable and stable process monitoring results.

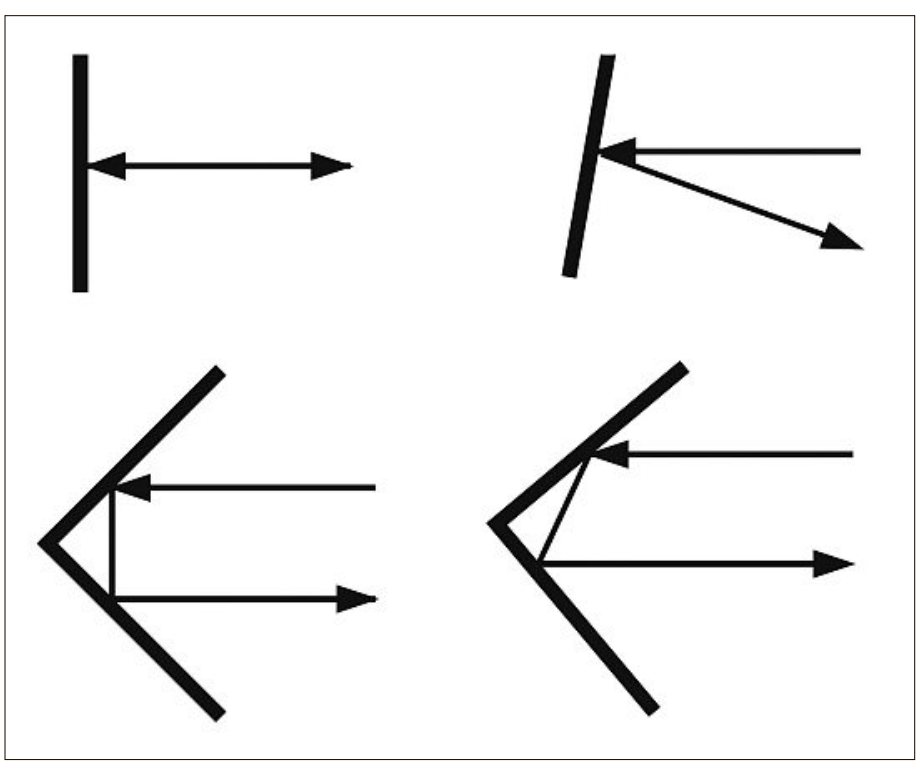

Fig. 3. Schematic view of a planar mirror vs a Cube Corner mirror. A small tilt in the flat mirror will reflect the light at a wrong angle. Cube Corner mirrors are not affected by tilts.

In an in-line measurement mode, the effect of heterogeneity is compensated further by the natural movement of the sample passing the spectral window of the probe. With FT-NIR even the presence of bubbles inside a reactor will not significantly disturb the measurement and will result only in a slightly decreased signal-to-noise ratio. The effect can be even further suppressed electronically by using a correlation mode inside the software which avoids the measurement whenever the signal is too low.

\subsubsection{No Chemicals, no Waste}

In contrast to wet chemical and chromatographic tools like GC or HPLC, the NIR method requires no chemical reagents and generates no waste or pollution.

\subsubsection{Long-range Data Transmission}

NIR spectra can be recorded directly in reactors and containers or tanks via fiber optic probes. The probes are directly mounted into the reactor transmitting the light over long distances ( $>100 \mathrm{~m}$ ) from and to the spectrometer, eliminating the need to install the spectrometer directly in the production or warehouse area.

\subsection{Probes}

Probes or measuring cells are at the heart of the process and designed to perform under harsh and fluctuating conditions. They are used to constantly assess the composition in real time and have to be very reliable. The selection of the materials for the probes is based on the (chemical) milieu and on the physical conditions (i.e. temperature and pressure) of the process. The probe construction materials include robust Hastelloy alloys, different stainlesssteel grades, or even extremely resistant titanium (Fig. 4). Different kinds of O-ring are used as sealing or even probes with welded optics are available.

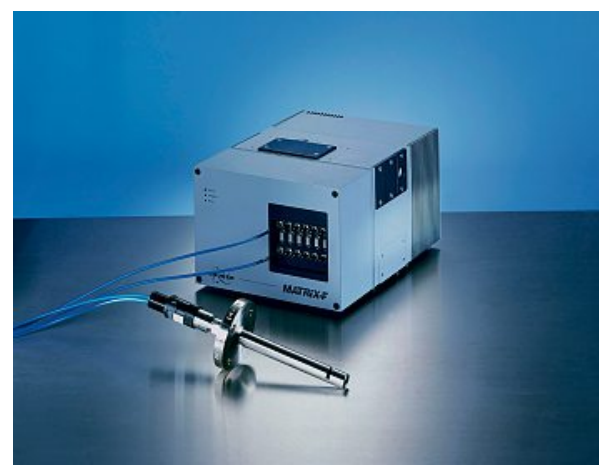

Fig. 4. Bruker Matrix-F with a transmission probe mounted on channel 1. The system can be equipped with up to six completely different probes for the monitoring of up to six completely independent and unrelated vessels.

The optical materials employed in the probes and measuring cells are often custom-made to match the process conditions. Quartz and sapphire do extremely well, thanks to their ultra-high degree of precision as well as their excellent chemical resistance. The probe required can be selected through a modular design approach and custom-made to fit the specific process conditions at hand.

\subsection{Multivariate Calibrations for FT- NIR Systems}

Compared to the classical univariate calibration, multivariate calibration methods use not only one spectral data point for the calibration, but the whole spectral range. The advantage of this type of calibration is the amount of spectral information accessible and processed, so that even minor differences in the sample spectra can be identified. ${ }^{[2]}$

Univariate calibration analysis is a method well known in any analytical laboratory and used for example also for FT-IR systems. Calibrating such a system 
requires the analysis of a set of calibration samples where known concentrations are plotted against the measured peak heights or areas. In order to analyze multicomponent samples, a characteristic signal for each component has to be identified and used for the calibration and subsequently the analysis. These signals must be well separated to lead to a reliable measurement. Univariate calibrations suffer from the following shortcomings:

- Outliers or perturbations caused by additional unknown components (contaminations) are not recognized because the concentration of the substance is determined by one spectral point only.

- Statistical fluctuations caused by detector noise influence directly the concentration values. Therefore, duplicates are required to assure the required accuracy.

Multivariate calibrations as used for FT-NIR systems take into account the spectral features over a wide range. Therefore, the analysis of overlapping spectral bands or broad peaks becomes feasible. The information contained in the spectra of the calibration samples will be compared to the information of the concentration values using a PLS (Partial Least Squares) regression. The method assumes that systematic variations observed in the spectra are a consequence of the concentration change of the components.

The advantages of multivariate calibration techniques are today undisputed.[2] By taking all the spectral information into account during the calibration process, the subsequent measurements can be carried out with high precision. Furthermore, it is possible to identify outliers reliably, and even to evaluate bands, which are massively overlapped with signals of other components, which often occurs in spectroscopy. The MDI-value (Mahalanobis-Distance) is constantly monitored and provides a measure whether the quality of the acquired spectrum is well represented by the calibration model. It is therefore a quality indicator providing a measure whether the calculated concentration result of a specific component is reliable or not.

The method development used for multivariate calibration is entirely different from the approach used in univariate calibration, although they both depend on a reference method. For univariate calibrations one normally tries, as described above, to find a signal that can be evaluated quantitatively without any overlapping from other spectral structures. In multivariate calibrations, larger spectral areas are combined intentionally. It does not matter whether the signals of various other system properties overlap in these areas. The combination which is best suited for the analytical method can be found reliably by testing all sensible combinations and performing a consecutive validation. Usually, no expert knowledge is required regarding the spectra of the pure substances which are to be analyzed.

\section{FT-NIR Spectroscopy Applied to Chemical Production Processes}

The fine chemicals industry, as mentioned earlier, still mostly relies on standard physico-chemical analytical methods (often installed in in-line mode) and off-line analysis especially for reaction monitoring and product quality analysis (see Fig. 5 - top section). However, the ever-increasing pressure on ecological and economical process sustainability requires the fine chemicals industry to step up to the next level. It is therefore essential to move towards the use of advanced process analytical techniques (PAT), ${ }^{[3]}$ with FT-NIR being one of the most promising tools. It can be applied to multi-component systems with concentrations ranging from $0.1-100 \%$, even for chemically similar substance structures.

At Firmenich, FT-NIR analysis has been evaluated in several (fed)-batch reaction and fractionation processes. For both process types off-line analysis is currently playing a pivotal role. The initial target for the fed-batch process was to replace off-line GC analysis leading to a process interruption and therefore to reduce the batch cycle time via an in-line FT-NIR-based process monitoring. In addition, transferring the information generated via the FT-NIR analysis to the DCS (Distributed Control System) would enable the production personnel to follow the fed-batch process in real time (see Fig. 5 - middle section).

In the case of the fractionation process, the objective was to improve the fraction collection process. So far, the fraction collection was based on a recipe and the individual fractions were then analyzed via off-line GC. Real-time and in-line FT-NIR analysis would allow off-line GC analysis and the recipe-based approach to be replaced with a more efficient and cost-effective control algorithm. This would also allow the work load on the central analytical lab to be reduced as well as trimming the number and the quantity of intermediate fractions having to be reprocessed and thereby liberating valuable production capacity.

The approach selected for the FT-NIR technology evaluation was to perform first a feasibility study on laboratory scale to assure that the tool could provide the required information. If successful and promising, the next step would be the implementation of the FT-NIR technology on production scale and to test the transfer of the calibration methods developed on lab scale (i.e. off-line) to production scale.

In a next phase and moving beyond process monitoring, the ultimate goal for the fed-batch reaction is using the new inline generated information to improve the product quality in the context of raw material variations and external disturbances including variations in the equipment performance. An additional ambition is to move from a recipe-based substrate feeding to a

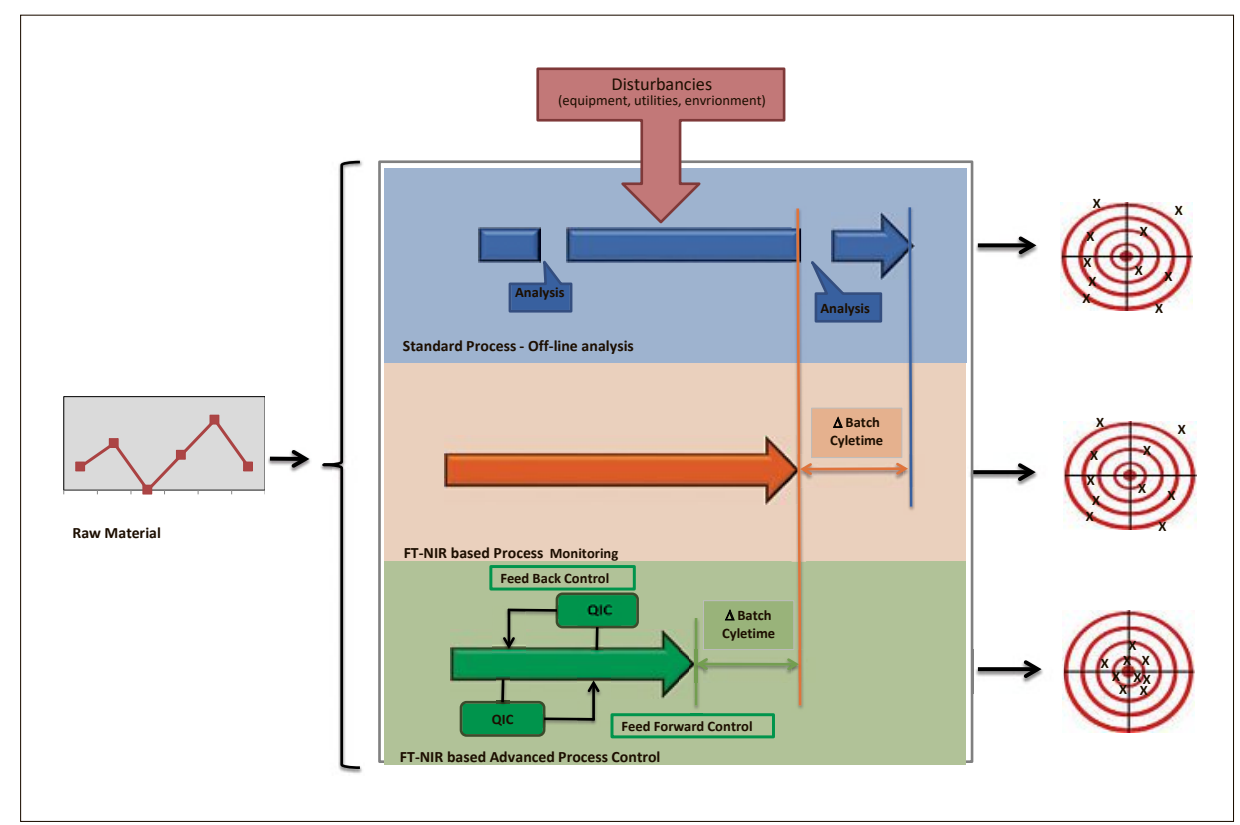

Fig. 5. Illustration of process optimization and process control options applying real-time process analysis via FT-NIR: Standard process: Off-line analysis - FT-NIR based process monitoring FT-NIR enabled closed loop advanced process control. On the right the precision of the predicted values is depicted. 


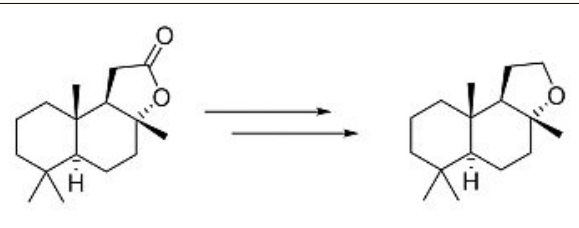

Scheme 1. Reaction Scheme: Sclareolide to Ambrox ${ }^{\circledR}$ Super transformation.

closed loop control of feed pumps applying both feed forward as well as feedback control (see Fig. 5).

\subsection{Feasibility Studies}

\subsubsection{Fed-batch Reaction - Laboratory Scale Feasibility Study}

The process selected for the technology assessment consisted of one of the steps of the chemical transformation represented by Scheme 1 .

In a first step, a fed-batch laboratory trial was carried out with an FT-NIR probe linked to a Bruker Matrix-F (see Fig. 4). The experimental set up is depicted in Fig. 6. A 1 liter laboratory reactor equipped with a $2 \mathrm{~mm}$ transmission probe was used for the initial assessment.

This setup allowed the laboratory-scale reaction to be followed in a continuous manner in real-time with a high resolution in time. From the collected spectral data, it was possible to identify distinct changes at specific wavelengths which in turn allowed trends to be extracted that reflect well the conversion of the substrate and the formation of the product (Fig. 7). This already demonstrated that FT-NIR could be used for a qualitative monitoring of the reaction.

However, in order to obtain quantitative information for the concentration of the substrate, product and side products a calibration via a primary analytical method had to be carried out. GC analysis was the reference method of choice as it had been used so far for the off-line process monitoring. The FT-NIR spectroscopy is typically an indirect analytical method unless samples with a known concentration and reflecting precisely process conditions can be prepared (e.g. via spiking). The initial calibration was performed with 15 samples taken during one laboratory run. These samples were then analyzed in off-line mode by GC based on an internal standard method supplemented with response factors. The GC data were then correlated to the FT-NIR spectra allowing a calibration to be obtained for the substrate conversions. As illustrated in Fig. 8 there was a very good fit between the effective measurements and the predicted value of the substrate concentration with only one outlier that did not fit the model well.

This initial laboratory scale investigation demonstrated the feasibility to moni-

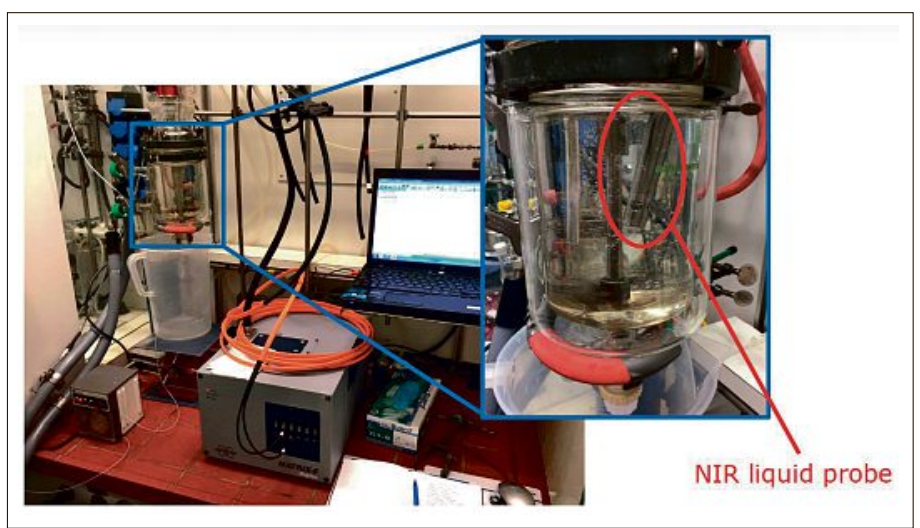

Fig. 6. Laboratory set up for the FT-NIR evaluation - 1 liter laboratory reactor with a $2 \mathrm{~mm}$ transmission probe installed and connected to a Matrix-F process spectrometer.

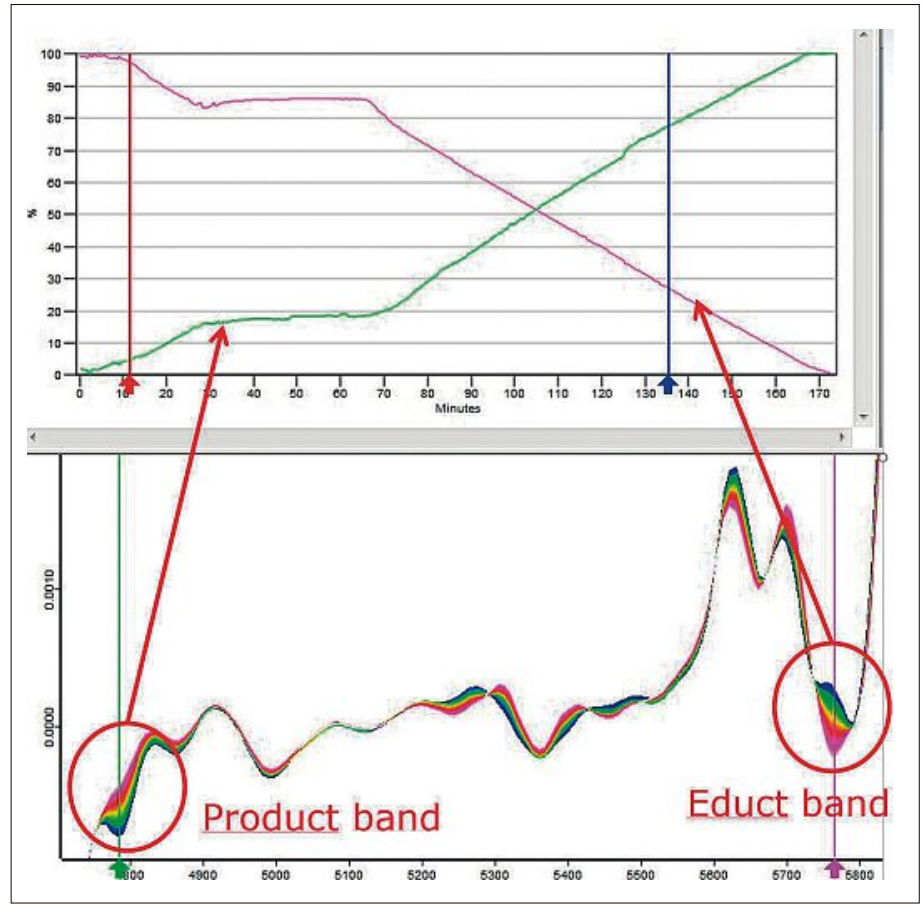

Fig. 7. FT-NIR spectra with an extraction of characteristic wavelengths representing the substrate conversion and production formation.

tor the targeted reaction via FT-NIR online and real-time analysis.

\subsubsection{Batch Fractionation - Feasibility Study}

Several approaches exist today to control and optimize batch fractionation processes with respect to the reflux ratio. However, the fractions are mostly still analyzed in off-line mode via GC or HPLC. Therefore, a fractionation recipe which is based on distillation time and/or distillate volume/mass has to be put in place. Based on the off-line analysis the fractions are then blended to afford the final product. Fractions not meeting the quality standard have to be reprocessed, leading to an important additional effort and a loss of production capacity. An in-line analysis in the reflux or the distillate line providing realtime composition information would allow to considerably optimize the fractionation process eliminating the need for off-line analysis. In addition, linked to a control algorithm, it would allow the number of reprocessed intermediate fractions to be reduced.

The batch fractionation selected for the assessment of the optimization potential via FT-NIR analysis is linked to a process featuring a partial conversion. The goal is to separate the residual substrate from a product and several side products. In a first step, the residual substrate has to be removed with a sufficient purity for direct recycling. In a next step, an intermediate fraction is separated before the product is removed and separated from the heavy side products.

Compared to the fed-batch process a different approach was selected for this feasibility study. Samples directly collected on production scale have been analyzed both by GC and FT-NIR in off-line mode. These two data sets have then been used to verify whether a correlation could be established for the different key compounds (e.g. residual substrate product and two side products). The first results were again very encouraging. Like in the first 
example a good correlation between the GC data and the FT-NIR spectra could be easily established as illustrated by Fig. 9 . This validated the suitability of FT-NIR analysis for in-line, real-time fraction process monitoring.

\subsection{FT-NIR Implementation Production Scale} laboratory scale feasibility studies and the promising process optimization potential it was decided to implement the FT-NIR technology on production scale for both investigated applications.

As depicted in Fig. 2, in both cases only the probe itself is installed in the hazardous environment, the light signal and data processing units (Matrix-F) have been installed in the electrical control room requiring optical fibers with a length of up to $150 \mathrm{~m}$.

\subsubsection{Fed-batch Process - FT-NIR Production Scale Implementation}

Due to space limitations, the FT-NIR probe had to be mounted from the reactor head, requiring a special $2.5 \mathrm{~m}$ long probe. Another challenge was the presence of bubbles and solids in the reactor.
Based on the positive results of the

In addition, it quickly became evident that it was not possible to establish one single calibration covering the entire concentration range with the accuracy required for the end point measurement. Therefore, two separate calibrations were developed, splitting the desired measurement range into two sections.

The first calibration covering the $100 \%$ $\rightarrow 20 \%$ range required a reliable indication with a limited precision. The goal for this process phase with the high secondary substrate feed rate was to remove the off-line GC analysis and the corresponding process interruption of 1 hour. Besides, the NIR evaluation results were intended to provide a real-time visualization of the reaction progress to allow the production personnel to take action if process deviations occurred.

The second part for the concentration range of $20 \% \rightarrow 0 \%$ with the low substrate feed rate, called for a precise measurement of the primary substrate in order to allow an accurate and reliable end point determination. Here again the target was to eliminate the off-line GC analysis for process follow-up to considerably reduce the batch cycle time by 1 hour and more. Whereas the set-up of the calibration for the phase 1

Fig. 8. Prediction vs True diagram, calibration of the substrate $\left(R^{2}=99.74\right)$, expected error $= \pm 1.78 \%$. Red diamond: Statistical outlier.

Fig. 9. Prediction vs True diagram, calibration of one compound $\left(R^{2}=99.99\right.$, expected error $= \pm 0.44 \%)$. Red diamond: Statistical outlier.
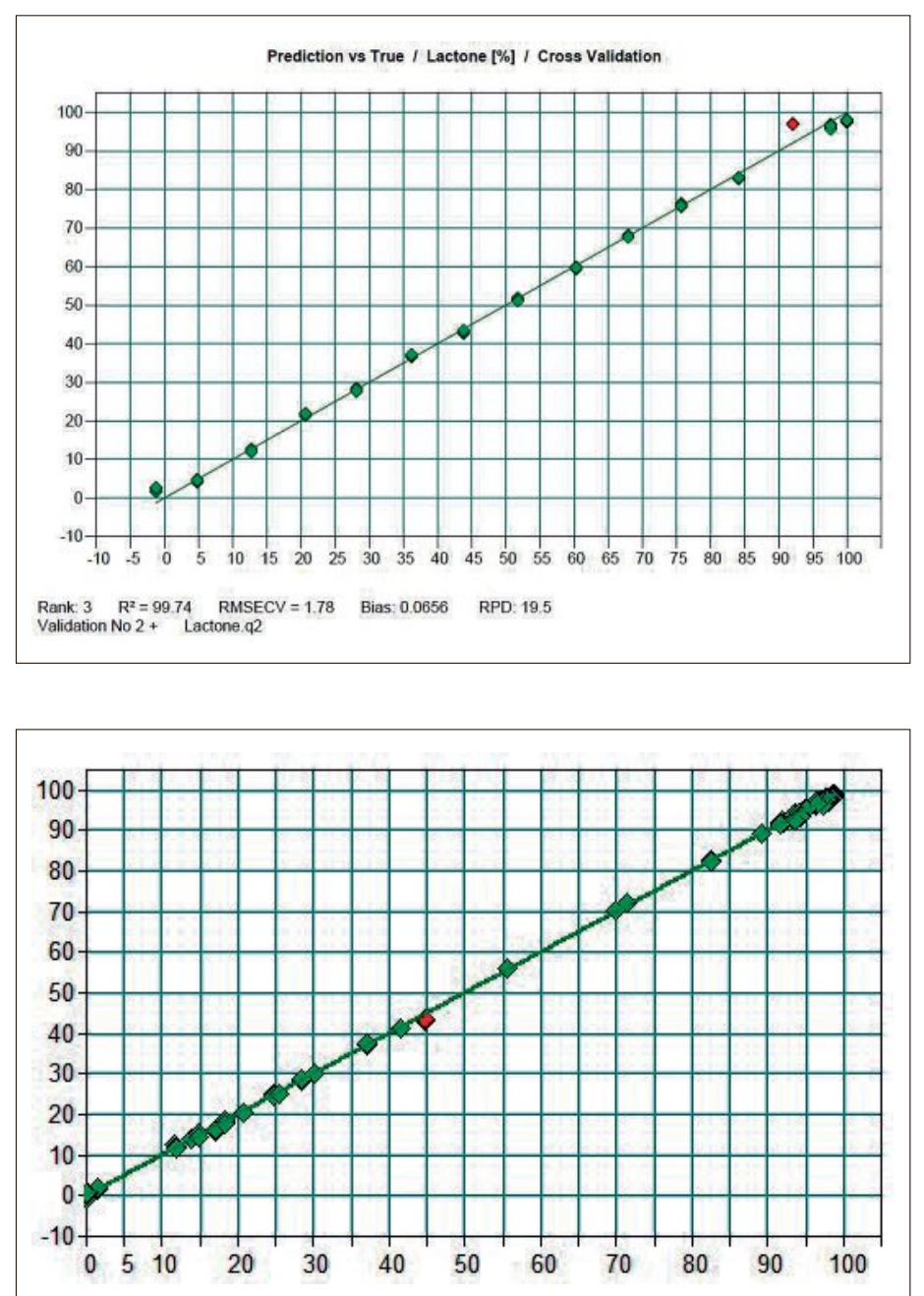

was rapid, the second part required several calibration iterations and a considerable number of data points to achieve the targeted accuracy. Besides an exact end point measurement, the real-time, in-line analysis will allow in the next phase to optimize the yield and to reduce substrate losses.

Finally, a stable and reliable calibration could be established for both sections as illustrated by Fig. 10. This allowed the batch cycle time to be reduced by more than two hours and consequently to increase the volumetric productivity of the reactor. In addition, the in-line analysis reduced the work load for the operators and for the central process analytical lab as no more sampling and off-line analysis was required. The FT-NIR allows also variations of the raw material and equipment performance to be identified. This real-time information enables the production teams to adjust the process leading to an improved yield and potentially a better product uniformity.

An FT-NIR method in place to monitor the process in-line and in a real-time mode will allow in a next phase to further reduce the batch cycle time by moving from a recipe-based substrate feed rate to a process-controlled addition rate (see Fig. 5). Precise determination of the initial and final substrate concentration will allow the process yield to be further optimized. In addition, reduced (minimal) residual substrate concentrations will simplify the following processing step.

\subsubsection{Batch Fractionation Monitoring}

For the in-line monitoring of the batch fractionation, the probe was mounted in the distillate line right after the condenser to minimize the time lag. The calibration established during the evaluation phase in 'off-line' mode on lab scale allowed the fractionation process to be monitored right after the installation of the FT-NIR probes. After some additional fine tuning, the FT-NIR delivered real-time concentration information which matched well the off-line GC analysis. Fig. 11 illustrates the evolution of a fractionation process based on FT-NIR analysis based on over 100 spectra. As indicated, the sum of all the concentration values was for most of the process close to $100 \%$. The somewhat lower total concentration during the second phase is explained by the fact that a few minor side products have not yet been integrated into the calibration. The dashed lines for the MDI values are for the entire process well below 1, highlighting that the FT-NIR measurements matched well the calibration model. Increasing MDI values in the range of the intermediate fraction (decreasing substrate $\mathrm{A}$ and increasing product and side products) indicate that some further fine tuning is required for this important process phase. 
The possibility to follow the batch fractionation in real-time will allow to move from a recipe-based process to a process controlled via in-line FT-NIR with the following benefits

- The number and size of intermediate fractions required to be reprocessed is reduced, thereby increasing the capacity of the production vessel.

- The quality of the recycled residual substrate but also of the product is improved.

- The work load for the analytical lab and the operation personnel is reduced.

All of this will lead to cost reductions translating into a fast investment payback.

\section{Summary and Outlook}

Analytical instruments for in-line and real-time measurement of physicochemical attributes (temperature, pressure, conductivity, $\mathrm{pH}, \mathrm{pO}_{2}$, etc.) are well established and a large range of instruments is available. However, instruments useful for the analysis of molecular attributes (i.e. concentrations of specific compounds and their evolution) are still scarce. FT-NIR is here one of the few possible choices. Feasibility has been demonstrated for many applications ranging from raw material inspection and validation, to process monitoring and quality control of intermediate as well as final products. In addition, the installation requires typically only a short downtime. Looking at the potential, it seems like FT-NIR is poised to play a crucial role in the process monitoring and visualization of fine chemical production processes. However, in spite of all the promising advantages presented in this article, it seems that the culture change is still happening at a rather slow pace.

The examples presented illustrate the potential to optimize (fed) batch and (batch) fractionation processes through the application of in-line analysis via FTNIR spectroscopy. FT-NIR has provided insight into reaction kinetics and even reaction mechanisms, including initiation mechanisms, substrate conversion, product as well as side product formation and optimal endpoint determination. Furthermore

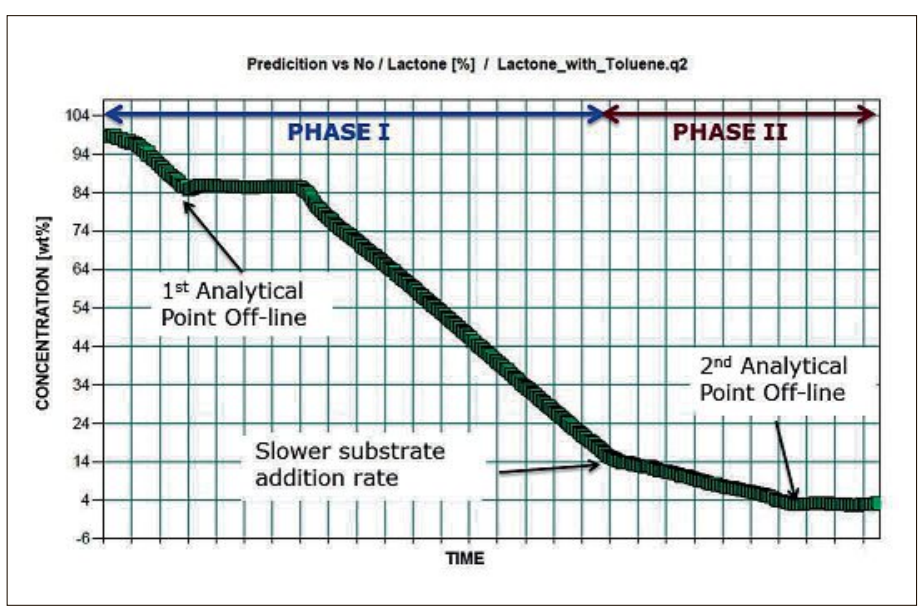

Fig. 10. Illustration of the visualization of substrate conversion via FT-NIR in-line, real time analysis.

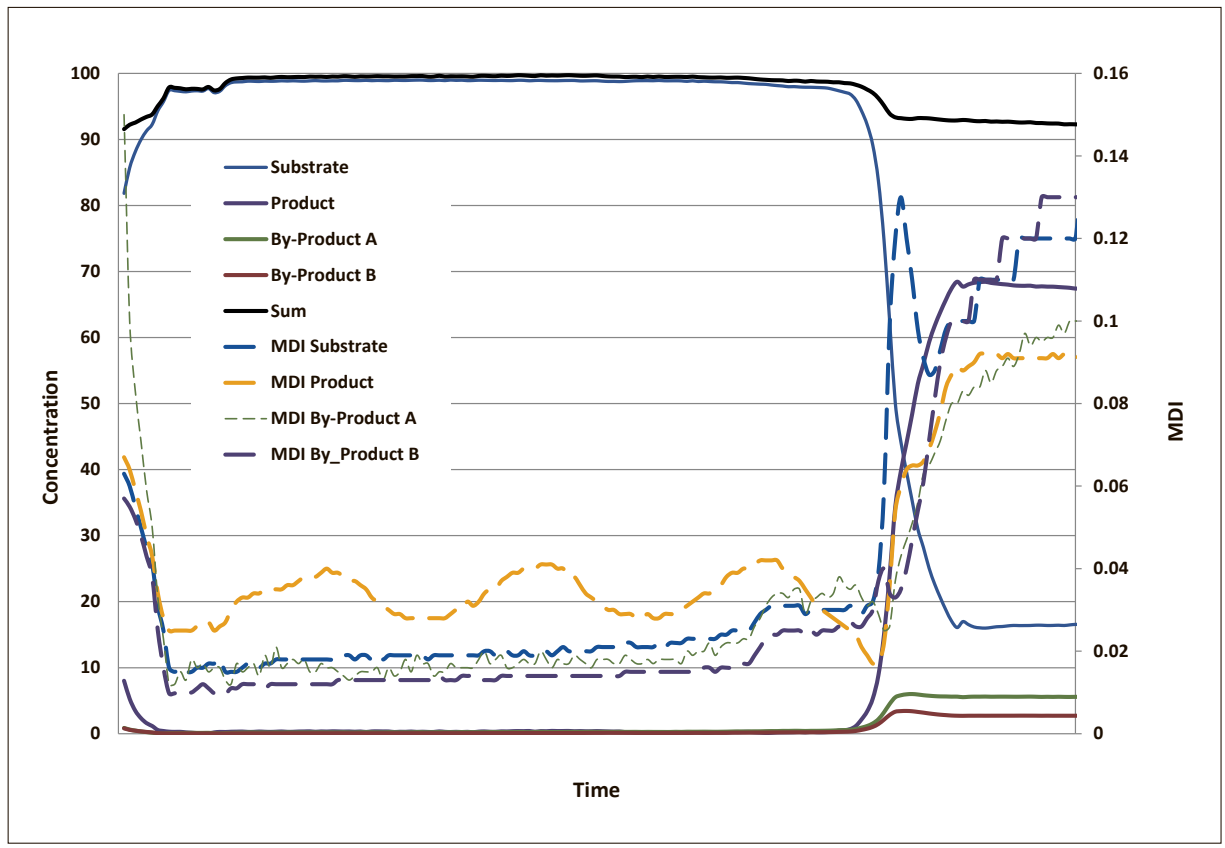

Fig. 11. Batch fractionation - FT-NIR measured evolution of critical substance concentrations.

FT-NIR helps to identify what is often referred to as a 'Golden batch'. More importantly FT-NIR provides a tool to reproduce the 'Golden Batch' reliably on production scale by following the corresponding trends of critical parameters.

The process gains allow typically for a process optimization, process innovation and ultimately for a very fast payback.

Received: January 31, 2018
[1] a) 'Near-Infrared Spectroscopy - Principles, Instruments, Applications', Eds H. W. Siesler, Y. Ozaki, S. Kawata, H. M. Heise, Wiley-VCH, Weinheim, 2002; b) B. H. Stuart, 'Infrared Spectroscopy: Fundamentals and applications', John Wiley and Sons, New York, USA, 2004.

[2] H. Martens, T. Naes, 'Multivariate Calibration', J. Wiley \& Sons, New York, 1989.

[3] M. Watari, Opt. Rev. 2010, 17, 317. 